\title{
New paradigms in the treatment of genitourinary malignancies: GU-ASCO Update 2014
}

\author{
Anil Kapoor, MD, FRCSC \\ Department of Surgery, Division of Urology, McMaster University, Hamilton, ON
}

Cite as: Can Urol Assoc J 2014;8(3-4Suppl2):S7. http://dx.doi.org/10.5489/cuaj.2010 Published online April 14, 2014.

W e are in the midst of a paradigm shift in the treatment of genitourinary malignancies. With the arrival of novel new agents, such as denosumab (Xgeva), abiraterone (Zytiga), cabazitaxel (Jevtana) and most recently enzalutamide (Xtandi) and radium-223 (Xofigo), we now have more options for the management of the castration-resistant prostate cancer (CRPC) patient. One puzzle is how these names are chosen - any novel agent starting with the letters " $\mathrm{x}$ " or " $z$ " has a high likelihood of having some activity in the CRPC patient! Our challenge now is how to best sequence these agents to achieve optimal outcomes in the CRPC patient, while at the same time being cognizant of fiscal responsibility. Should all CRPC patients be offered novel agent $X$ or $Z$ ?

This year at the annual GU-ASCO conference, landmark studies in the treatment of advanced prostate cancer were presented and are summarized in this congress report. The highlights of the Multinational Phase 3, Randomized, Double-Blind, Placebo-Controlled Efficacy and Safety Study of Oral MDV3100 in Chemotherapy-Naive Patients With Progressive Metastatic Prostate Cancer Who Have Failed Androgen Deprivation Therapy (PREVAIL) study of enzalutamide in the chemotherapy-naïve CRPC patient are summarized in detail. Updates on abiraterone, denosumab and radium-223 in the advanced prostate cancer patient are presented, as well as recent data on sequencing of these agents.

In addition to a prostate cancer update, we summarize the recent data on advanced kidney cancer from Genitourinary Cancers Symposium (GU-ASCO) congress. Sequencing studies with tyrosine-kinase inhibitor (TKI) therapy are presented, as well as highlights from the IMDC database analysis. In the current era of targeted therapy, data supporting the beneficial effect of cytoreductive nephrectomy are presented.

Finally, this congress report summarizes some of the Canadian research highlights presented at GU-ASCO, including highlights from the International mRCC Database Consortium (IMDC) and the Canadian Kidney Cancer Information System (CKCis) database analysis, and clearly illustrates the high-level oncology research coming out of our great Canadian research institutions.

We hope you enjoy it and find the information useful!

Competing interests: Dr. Kapoor is a member of the Speakers bureau for, and has received grants and honoraria from, Pfizer Oncology, GSK Oncology, Novartis Oncology, Astellas, Janssen, and Amgen. He has also participated in clinical trials within the past 2 years with NCIC, Pfizer, GSK, Novartis, Astellas, Janssen, and Amgen.

Correspondence: Dr. Anil Kapoor, Professor of Surgery (Urology) and Chair, Genitourinary Oncology Program, Juravinski Cancer Centre, McMaster University, St. Joseph's Hospital, 50 Charlton Ave. E., Hamilton 0N L8N 4A6; akapoor@mcmaster.ca 